\title{
Experimental validation of an impact off-resonance energy harvester
}

\author{
G. Martinez-Ayuso, M.I. Friswell ${ }^{a}$, H. Haddad Khodaparast, and S. Adhikari \\ College of Engineering, Swansea University, Bay Campus, Fabian Way, \\ Swansea SA1 8EN, UK
}

Received 30 September 2018 / Received in final form 18 January 2019 Published online 23 August 2019

\begin{abstract}
Most piezoelectric energy harvesting research has focused on developing on-resonance harvesters that work at low frequencies, even though higher frequencies can generate more power. In addition, conventional resonant harvesters have low efficiency when the excitation frequency is away from resonance. Using mechanical impacts has the potential to improve the overall harvested energy since high frequencies are excited during impacts. Also, the presence of impacts reduces the influence of the base excitation frequency and the requirement to exactly match the resonance frequency. To take advantage of the higher frequency response, an impact energy harvester is designed and validated experimentally. The harvester consists of a cantilever beam with a piezoelectric patch attached to its base which impacts with a stiff object. The harvester is modelled using finite element analysis and a Hertzian contact law. The model is tested and validated in the laboratory using an in-house manufactured demonstrator. Good agreement with the experimental data is obtained, setting the basis for future optimisation of the harvester geometry and piezoelectric properties.
\end{abstract}

\section{Introduction}

The harvesting of energy from the environment has become important in powering systems where a wired system or the use of batteries is not appropriate, such as remote sensing systems. Piezoelectric materials have played an important role scavenging energy from vibrations in the environment for over a decade. Different authors have investigated the application of these materials under different conditions of excitation. For instance, Erturk and Inman [1] studied the performance of a cantilever beam energy harvester $(\mathrm{EH})$ under harmonic base excitation. The importance of a correct optimization of the electrical circuit attached to the piezoelectric patch was highlighted. Adhikari et al. [2] studied random excitations using a stochastic approach on a single-degree-of-freedom model. The importance of a low mechanical damping and high electromechanical coupling to obtain the maximum power was shown. Also, different geometrical configurations have been analysed such as beams [1] and plates $[3,4]$.

a e-mail: m.i.friswell@swansea.ac.uk 
Linear energy harvesters are based on tuning the natural frequency of the harvester to match the excitation frequency [2], so that the amplitude of the vibration, and hence the power output, is maximum. The excitation frequency is then a constraint in the design of the energy harvester. Normally, natural sources of vibration, such as wind or waves, or machinery are used to excite the energy harvester. These source of vibration are normally low in frequency $(<100 \mathrm{~Hz})$ and therefore, the first vibration mode of the harvester is designed to match the excitation frequency. However, higher modal frequencies might generate more energy than lower ones.

Impacts have been proved to be an efficient way to excite higher frequencies using low frequency excitation. This conversion mechanism allows the conversion of low frequencies to high frequencies $(1 \mathrm{kHz}-1 \mathrm{MHz})$ [5] depending mainly on factors such as the impact stiffness or contact duration. Using this principle, some authors have developed different models of impact energy harvesters.

One of the first contributions to the field of impact energy harvesting was made by Umeda et al. [6], where the dynamic system was solved analytically using an equivalent electrical circuit. The importance of the quality coefficient and the existence of an optimal resistance was presented. Cavallier et al. [5] studied an experimental rotational impact piezoelectric energy harvester. This harvester used a silicon cantilever beam as vibrating energy storage element and mechanical shocks as the excitation source. The shock excitation generates a higher voltage output. Renaud et al. [7] investigated the performance of a cantilever beam EH with impact. This harvester was modelled as a single-degree-of-freedom system, the dynamic equations were solved by establishing an equivalent electrical model, and the impact was applied as an instantaneous velocity load at the tip. This model gives a good insight of the dynamic behaviour of an EH, although the equivalent electrical circuit does not allow the detailed modelling of the contact.

Jacquelin et al. [8] modelled the impact between two cantilever beams and a seismic mass using the anti-oscillator approach. The dynamic equations were solved for a limited number of degrees of freedom using the Rayleigh-Ritz procedure and the Hertzian contact law was included [9]. The authors concluded that the maximum power obtained is due to the transient (impact) regime; this power is much higher than that obtained in the linear steady state (harmonic excitation) although the steady state power is more constant with time. Gu and Livermore [10] modelled, simulated and tested experimentally a piezoelectric energy harvester impacting against a beam, and highlighted a much higher efficiency of the impact energy harvester compared to the linear counterpart. The shift towards higher frequencies was shown and the importance of mechanical damping in order to improve the power obtained is presented. However, in that paper the effect of the base excitation frequency and the resistance was not assessed.

Vijayan et al. [11] investigated two piezoelectric cantilever beams impacting with each other. The contact is modelled using a linear spring with high stiffness. In this harvester, the power is highly sensitive to the clearance and thickness ratio. The presence of impacts generates more peaks in the frequency response at different frequencies which means more modes are excited and more power can be harvested. Martinez-Ayuso et al. [12] considered the use of tailored material properties to enhance the performance of the impact harvester. A composite porous piezoelectric material was used in order to improve the performance. The low significance of the resistance and the good performance of the energy harvester away from resonance was shown.

This paper investigates the dynamics of impact harvesters under base excitation, based on simulated results that are validated with experimental data. The paper is divided in six sections. The state of the art in impact energy harvesting is given initially. The details of the experimental energy harvester and the linear model validation are then described, followed by the model formulation including the assumptions and 


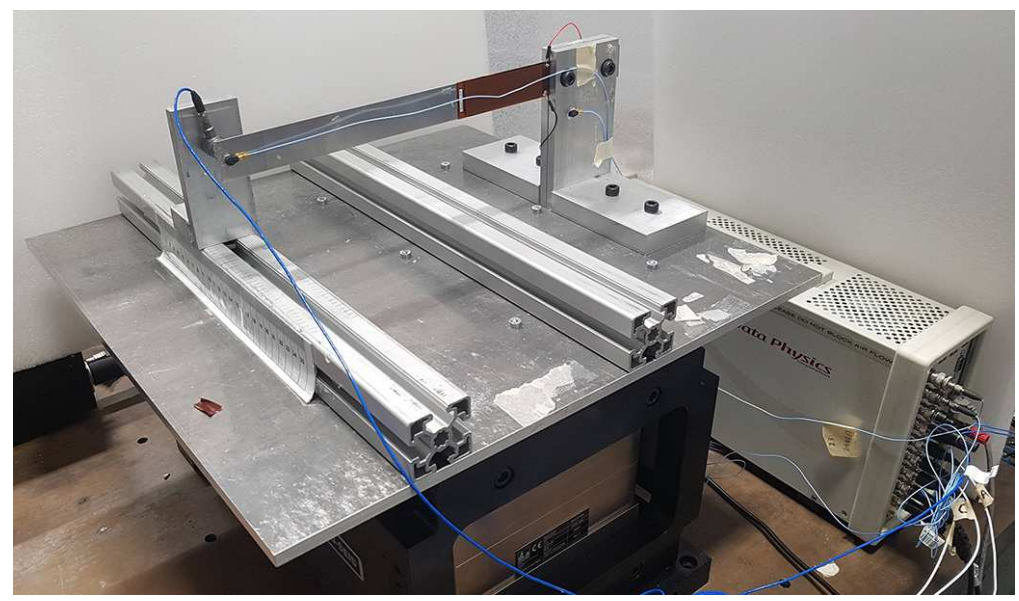

Fig. 1. The experimental setup.

impact law. The results of the simulations and the experiments then follow. Finally, the results are discussed and conclusions are drawn.

\section{The linear harvester: test setup, modelling and validation}

The experimental device is shown in Figure 1 and consists of a beam mounted in a cantilever configuration on a shaking table to provide base excitation. A single Macro Fiber Composite (MFC) piezoelectric patch (Smart Material type M8528-P2) is bonded to the beam close to the root (the spacing between the support and the patch is approximately $9 \mathrm{~mm}$ ). Impacts are applied to the beam approximately 10 $\mathrm{mm}$ from the free end using a steel tip from an impact hammer used in modal testing which includes a force transducer to measure the reaction force. The steel tip is very stiff with a maximum excitation bandwidth of approximately $7 \mathrm{kHz}$, although the beam flexibility will reduce the actual bandwidth significantly. Table 1 gives the geometry and properties of the beam and piezo patch. Figure 2 shows the top view of the beam with the direction of the base excitation and highlighting the gap between the undeformed beam and the steel tip at the contact area. Figure 3 shows the circuit to measure the output from the piezo patch, which also includes a load resistor.

The model of the linear energy harvester is now described. The beam is very thin and hence is modelled using standard Euler-Bernoulli beam elements. The beam stiffness $(E I)$ is increased in the region of the piezo patch to allow for the stiffening effect. The mass of the MFC patch is neglected since the patch is light and close to the root of the beam. Only one MFC piezoelectric patch is added to a beam and hence forms a unimorph configuration. The moment about the beam neutral axis produced by a voltage $V$ across the piezoelectric layers $[13,14]$ may be written as

$$
M_{\Lambda}(s, t)=\gamma_{p} V(t)
$$

where the constant $\gamma_{p}$ depends on the geometry, configuration and piezoelectric device and $s$ denotes the location of the end of the piezo patch nearest the tip. The subscript $p$ denotes piezoelectric material properties. For a unimorph with piezoelectric layers in the 31 configuration, with thickness $h_{p}$, width $b_{p}$, the piezoelectric coupling constant 
Table 1. Parameters for the beam example.

\begin{tabular}{lll}
\hline & Beam & Piezo Patch \\
\hline Length & $315 \mathrm{~mm}$ & $85 \mathrm{~mm}$ \\
Width & $30 \mathrm{~mm}$ & $30 \mathrm{~mm}$ \\
Thickness & $0.98 \mathrm{~mm}$ & $0.3 \mathrm{~mm}$ \\
$E$ & $65 \mathrm{G} \mathrm{Pa}$ & $30.336 \mathrm{GPa}$ \\
$\rho$ & $2750 \mathrm{~kg} / \mathrm{m}^{3}$ & \\
$d_{31}$ & & $-170 \mathrm{pC} / \mathrm{N}$ \\
$C_{P}$ & & $177 \mathrm{nF}$ \\
\hline
\end{tabular}

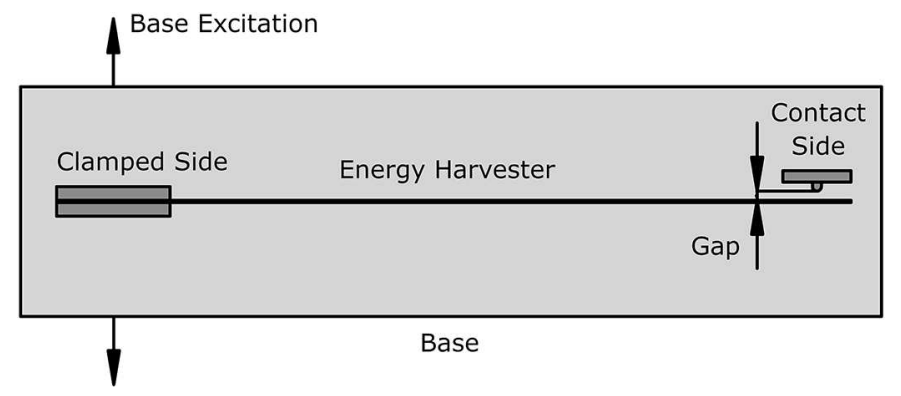

Fig. 2. Top view of the beam with the excitation and contact.

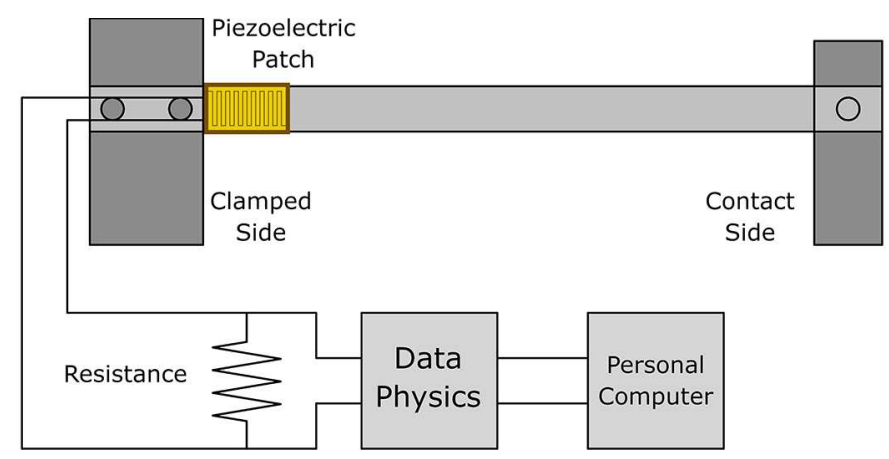

Fig. 3. Schematic of the experimental setup.

is

$$
\gamma_{p}=E_{p} d_{31} b_{c}\left(h+\frac{h_{c}}{2}-\bar{z}\right)
$$

where $h$ is the thickness of the beam, $d_{31}$ is the piezoelectric constant, and $\bar{z}$ is the effective neutral axis [15]. These expressions assume a monolithic piezoceramic actuator perfectly bonded to the beam; Bilgen et al. [16] considered the effect of the structure of a Macro-Fiber Composite (MFC) on the coupling coefficient, and also the effect of the bond and glueing layers.

In the electrical circuit the piezoelectric patch is modelled as a capacitor, $C_{p}$, in series with a load resistor, $R$. Then

$$
C_{p} \dot{V}+\frac{V}{R}+\gamma_{p}\left(\dot{\theta}_{2}-\dot{\theta}_{1}\right)=0
$$


Table 2. Natural frequencies and damping ratios for the linear beam.

\begin{tabular}{lll}
\hline $\begin{array}{l}\text { Model natural } \\
\text { Frequencies (Hz) }\end{array}$ & $\begin{array}{l}\text { Measured natural } \\
\text { Frequencies (Hz) }\end{array}$ & $\begin{array}{l}\text { Measured damping } \\
\text { Ratios (\%) }\end{array}$ \\
\hline 8.66 & 9.01 & 0.194 \\
50.3 & 49.7 & 0.925 \\
140.9 & 133.2 & 0.864 \\
278.9 & 262.8 & 0.359 \\
457.6 & 436.2 & 0.126 \\
681.9 & 651.3 & 0.274 \\
960.5 & 918.1 & 0.160 \\
\hline
\end{tabular}

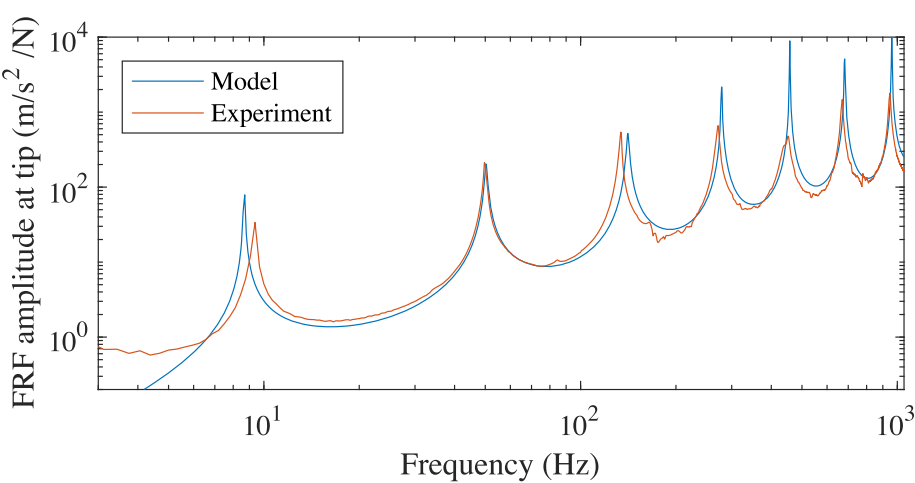

Fig. 4. Frequency response function for the acceleration at the free end.

where $\theta_{1}$ and $\theta_{2}$ are the slopes of the beam at the ends of the piezoelectric patch.

Measurements on the physical beam were obtained using impact excitation approximately $15 \mathrm{~mm}$ from the root of the beam with the table fixed. The response was measured using an accelerometer near the tip and also the output from the piezo patch in an open circuit configuration. Table 2 gives the measured and modelled natural frequencies and Figures 4 and 5 show the frequency response functions for the tip acceleration and the piezo output. The form of the responses closely match, although there are clearly some minor parameter differences. Typical modelling approximations include the stiffness of the clamped support, the stiffness and electromechanical properties of the MFC patches, and the effect of the adhesive layer used to bond the patch to the beam. Although the model could be further refined to improve the correlation, the model is sufficiently accurate to enable the interpretation of the experiments with impact, and will allow future optimisation studies.

\section{Simulation of the impact harvester}

The cantilever beam harvester is now supported on the shaker table. The rotation at the clamped end of the beam is still constrained to be zero, but now the displacement is free and a mass of $12 \mathrm{~kg}$, which approximates the moving mass of the shaker table, is applied to this degree of freedom. The shaker has a centring spring and this is modelled with a grounded spring to give a rigid body mode of the shaker of $1 \mathrm{~Hz}$, which is well below the beam natural frequencies. The shaker force is then applied to the degree of freedom representing the table mass. This approach is adopted rather than enforcing a base displacement or acceleration because the measured base acceleration 


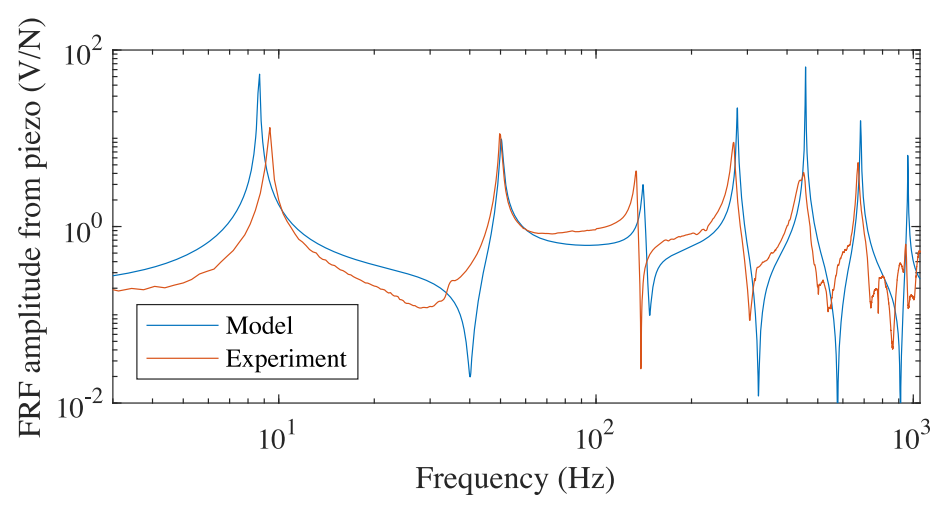

Fig. 5. Frequency response function for the MFC voltage.

is affected by the reaction force of the beam during impacts. This approach then enables the simulations to validate the experimental results.

The contact is modelled as a non-linear spring located at the contact point and is only active while the contact occurs. This force is based on the contact Hertz law:

$$
F_{c}=-k_{c} \delta^{3 / 2} \quad \text { if } \quad \delta>0
$$

where $\delta$ is the penetration or gap function, defined by the distance between the contact point on the beam and the steel tip, and $k_{c}$ is the stiffness of the contact. The gap between the undeformed beam and the steep tip is defined as $g$ and hence $\delta=x_{c}-g$, where $x_{c}$ is the displacement of the beam at the contact.

The equations of motion of the beam are then

$$
\mathbf{M} \ddot{\mathbf{q}}+\mathbf{C} \dot{\mathbf{q}}+\mathbf{K q}-\gamma_{p} \mathbf{B}_{p} V-F_{c} \mathbf{B}_{c}=\mathbf{B}_{s} f_{s}(t)
$$

where $\mathbf{q}$ are the beam degrees of freedom, and $\mathbf{M}$ and $\mathbf{K}$ are the mass and stiffness matrices. The vector $\mathbf{B}_{p}$ picks out the rotational degrees of freedom at the ends of the piezoelectric patch, $\mathbf{B}_{c}$ picks out the contact degree of freedom, and $\mathbf{B}_{s}$ picks out the shaker degree of freedom. $f_{s}$ denotes the shaker force, which in the examples will be sinusoidal. $\mathbf{C}$ is the damping matrix; in the simulations the model will be reduced to the first eight modes and modal damping will be applied using the measured damping ratios.

The electrical equation is

$$
C_{p} \dot{V}+\frac{V}{R}+\gamma_{p} \mathbf{B}_{p}^{\top} \dot{\mathbf{q}}=0
$$

\section{Simulated results for the impact harvester}

The model is reduced to the first eight modes of the beam and formulated in state space. The contact stiffness coefficient is assumed to be $k_{c}=1000 \mathrm{~N} / \mathrm{m}^{3 / 2}$ and the gap is $1 \mathrm{~mm}$. The model is integrated using ode 45 for $20 \mathrm{~s}$, and the last $10 \mathrm{~s}$ is used for the analysis after the transients have decayed. The average power harvested between times $T_{1}$ and $T_{2}$ is estimated as

$$
P_{\text {ave }}=\frac{1}{T_{2}-T_{1}} \int_{T_{1}}^{T_{2}} \frac{V(t)^{2}}{R} \mathrm{~d} t .
$$




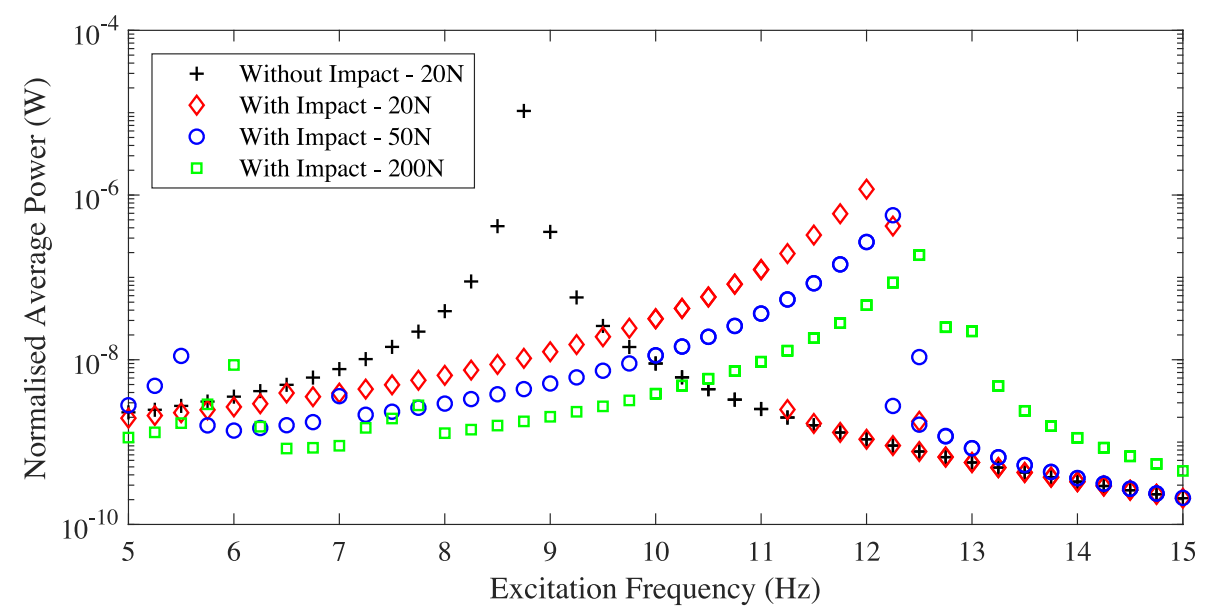

Fig. 6. The average power generated by impact.

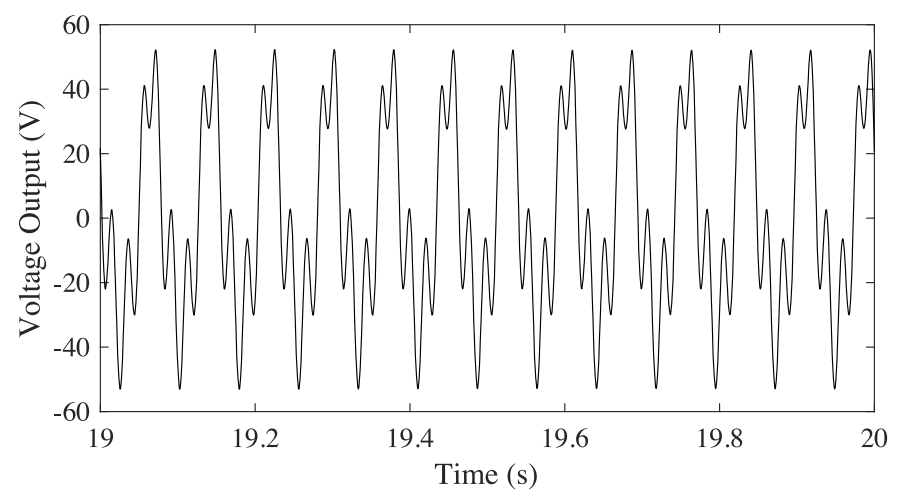

Fig. 7. The voltage output with impact for an excitation of $13 \mathrm{~Hz}$ and $200 \mathrm{~N}$.

When comparing the results at different frequencies and force amplitude we need to normalise the average power estimates for the different levels of energy input. Here we normalise the estimated average power by the square of the force applied; for a linear system this metric would not change with the force level applied. Note that the RMS base acceleration will be excitation frequency dependent in this case because we model a force applied to the shaking table. Figure 6 shows the estimated power as the excitation frequency varies for the linear case, and also the case with impact for three excitation levels. The load resistance is fixed at $1 \mathrm{M} \Omega$. The linear harvester performs best near resonance, as expected. The impact has caused a hardening response causing the resonance frequency to increase. Jumps in the estimated power are clearly visible; these results have been obtained by stepping up in frequency, ensuring a continuous beam response during the frequency change, to maintain the solution on a particular solution branch for as long as possible. The frequency is then swept down through the resonance region. Multiple solutions are apparent for some excitation frequencies near the jump region.

For the cases with impact, higher modes can be excited. The power generated for an excitation of $13 \mathrm{~Hz}$ and $200 \mathrm{~N}$ is relatively large; Figure 7 shows the voltage output for the last $1 \mathrm{~s}$ of the simulation and clearly shows that the second mode is excited at approximately four times the excitation frequency. 


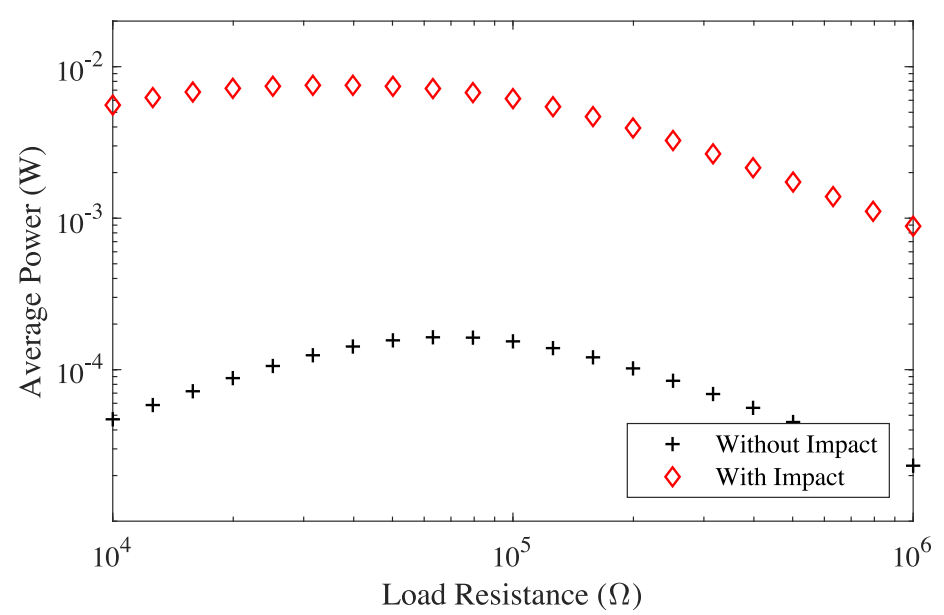

Fig. 8. The effect of load resistance on average power generated, with and without impact, for an excitation of $13 \mathrm{~Hz}$ and $200 \mathrm{~N}$.

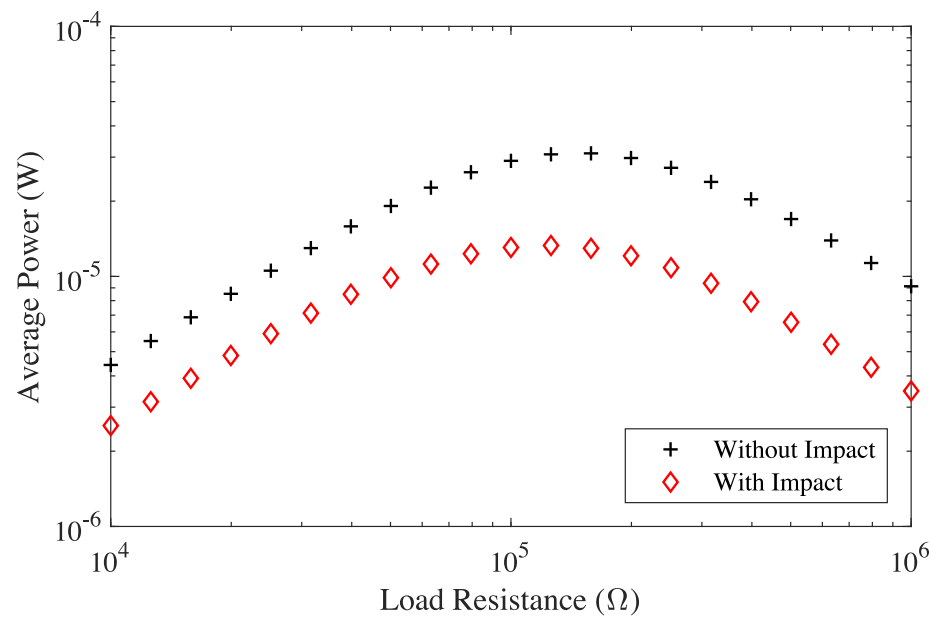

Fig. 9. The effect of load resistance on average power generated, with and without impact, for an excitation of $6 \mathrm{~Hz}$ and $50 \mathrm{~N}$.

Figures 8 and 9 show examples of the effect of the load resistance for two different excitation cases. For frequencies close to the resonance for the impact case the power generated by the impact harvester is larger. Furthermore the optimum load resistance is slightly smaller than for the linear case. Below the linear resonance the linear harvester performs better than the impact harvester. Once again, the optimum load resistance is slightly smaller for the impact case.

\section{Experimental results for the impact harvester}

The test rig was excited with a sinusoidal force and various load resistors were connected across the piezoelectric patch. After the transients had decayed the base acceleration, tip acceleration, piezoelectric voltage and contact force were measured at a sample rate of $1536 \mathrm{~Hz}$ for approximately $85 \mathrm{~s}$. Figures 10 and 11 show effect 


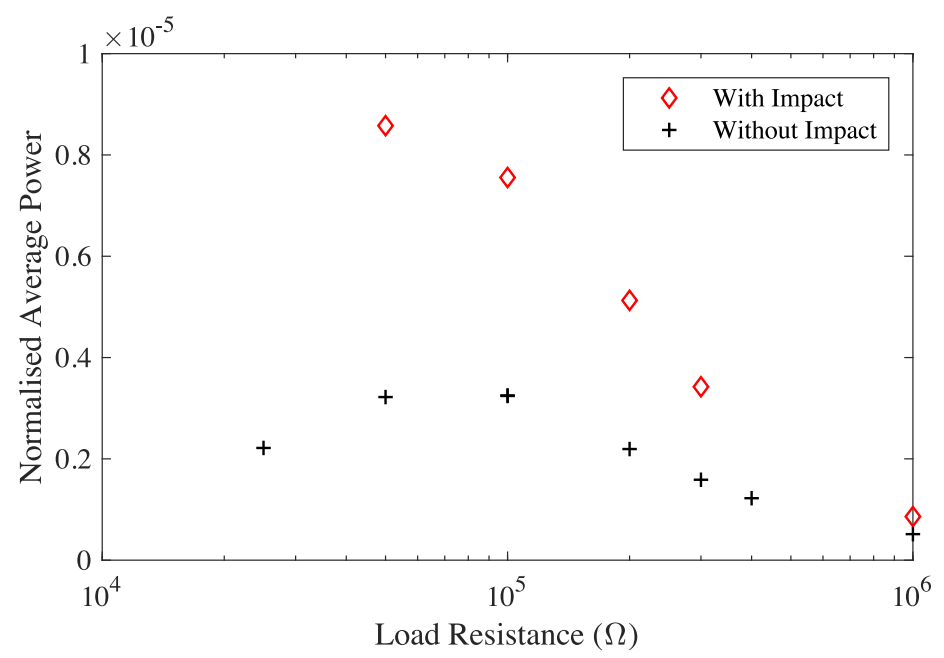

Fig. 10. The effect of load resistance on average power generated, with and without impact, for the experiment with excitation at $13.5 \mathrm{~Hz}$.

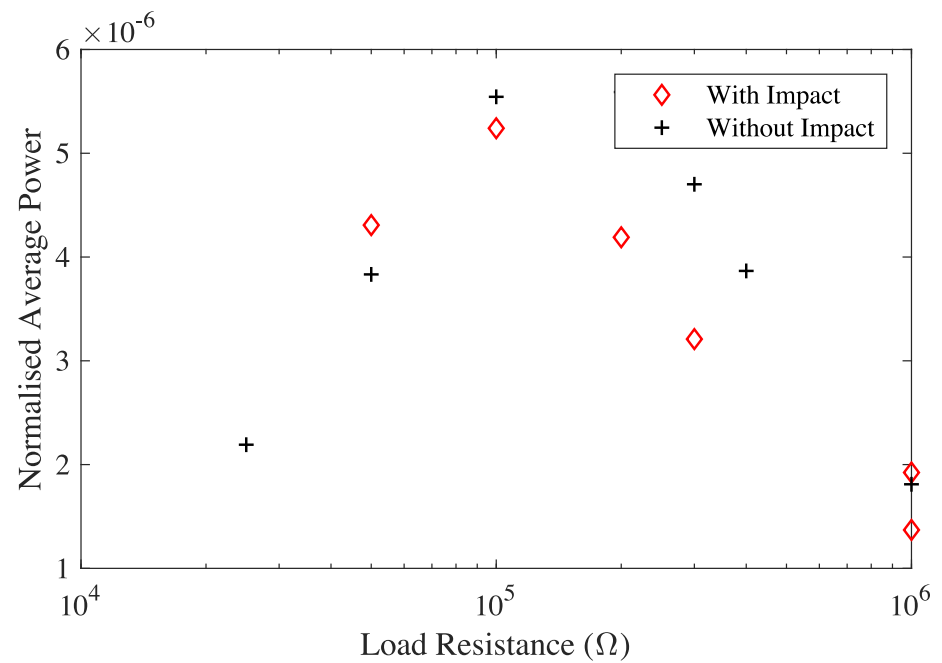

Fig. 11. The effect of load resistance on average power generated, with and without impact, for the experiment with excitation at $6 \mathrm{~Hz}$.

of the load resistance for two different excitation cases, above and below the first natural frequency of the linear system. Here the power is normalised by the average base acceleration squared. The experimental results show the same trends as the simulated results, namely that close to the linear resonance the linear harvester performs better and above the linear frequency, close to the nonlinear resonance frequency, the impact harvester performs better. The optimum load resistance is also slightly lower for the impact harvester.

The simulated results showed that the nonlinear resonance had a jump in the frequency response with multiple solutions. Figure 12 shows the experimental voltage response at $13.5 \mathrm{~Hz}$ with the $100 \mathrm{k} \Omega$ load resistor, and shows the beam jumping between two different solutions. This may be highlighted by calculating the FFT of the voltage for different periods of time covering different solutions, as shown in 


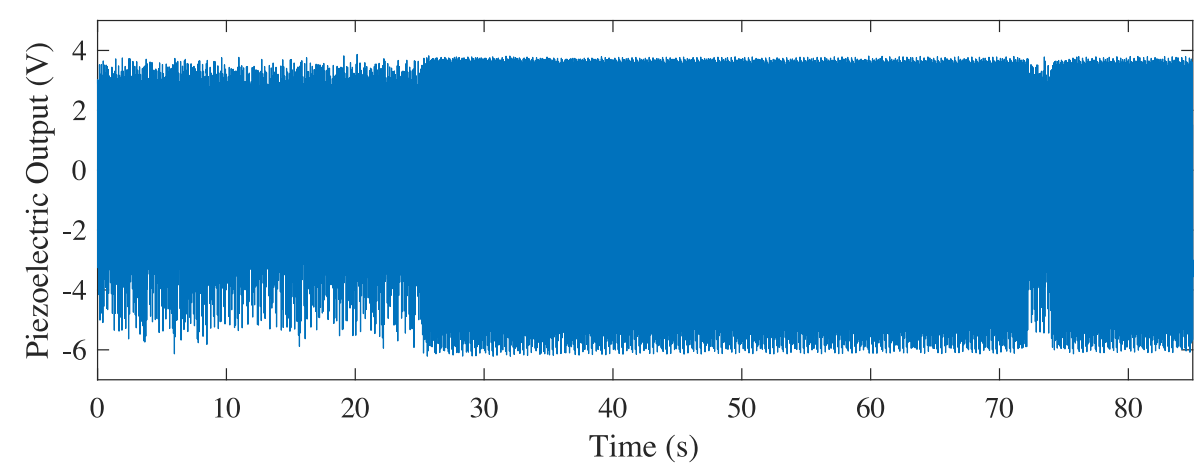

Fig. 12. The voltage time response, for the experiment excited at $13.5 \mathrm{~Hz}$.
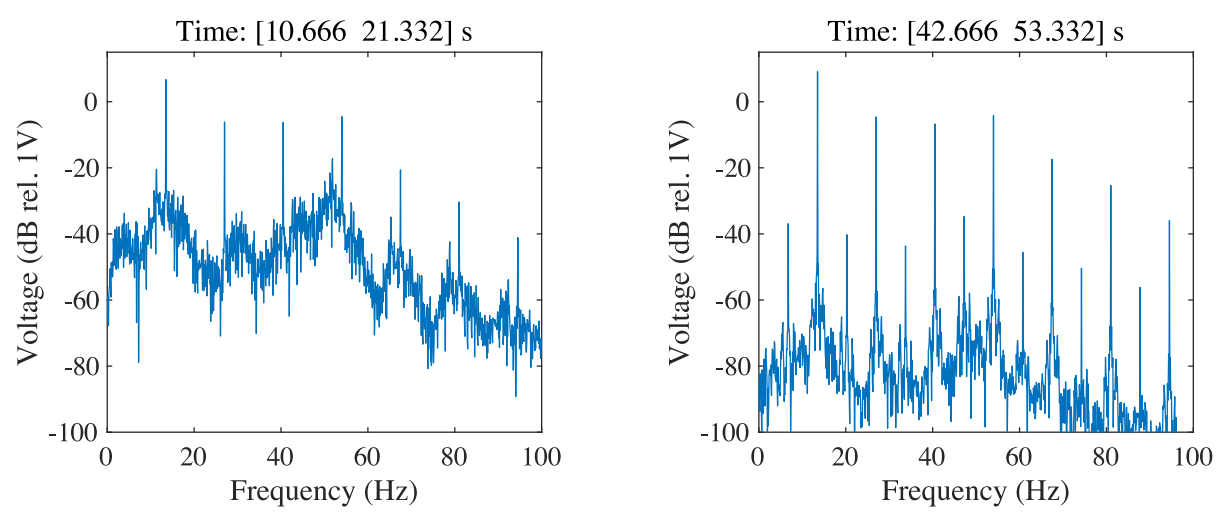

Fig. 13. The FFT of the voltage response at different times, for the experiment excited at $13.5 \mathrm{~Hz}$.

Figure 13. Clearly shown in both FFTs is the excitation frequency and its harmonics, which highlights that an essentially periodic response is dominant. The component at four times the excitation frequency (i.e. $54 \mathrm{~Hz}$ ) is the second highest component because this is close to the second beam natural frequency. However for the response shown on the right, there are also significant responses at half the excitation frequency and its harmonics. There is limited excitation of the beam resonances although for the response shown on the left there are noisy peaks with low amplitudes around $12 \mathrm{~Hz}$ and $50 \mathrm{~Hz}$. The average power generated in the time intervals shown in Figure 13 are $31.1 \mu \mathrm{W}$ and $48.1 \mu \mathrm{W}$ for the solutions corresponding the left and right plots; the right solution does have higher amplitudes at the higher frequencies.

\section{Discussion and conclusions}

This paper has investigated a vibration energy harvester consisting of a base excited beam with piezoelectric patch impacting on a stiff support near the tip of the beam. The benefits suggested are that the impacts would excite the higher modes of the beam, leading to the generation of higher levels of energy from the piezoelectric transducer. The excitation frequency must be reasonably close to the first resonance frequency, otherwise the response of the beam would be insufficient for impact to occur for this system. The excitation frequency and the low damping typical of beam structures (and required for efficient energy harvesting) means that impacts cannot 
be considered as isolated, where the beam response decays between impacts. Thus the typical beam response is periodic, often at the excitation frequency although subharmonics can be present. This means that the transient response at higher modes is generally not present, and hence the higher modes are often not strongly excited. However, if an harmonic of the excitation frequency matches one of the higher modes, then the response at this harmonic will be increased. But overall the effectiveness of this type of impact harvester to excite the higher modes is very limited.

The main effect of the impact is to stiffen the system and hence increase the resonance frequency. However the level of power generated at the resonance frequency is similar to the linear case. The stiffening level and hence resonance frequency increases with the amplitude of the excitation, because the time in contact increases. A similar effect would occur with changing the gap between the undeformed beam and the contact, and this could possibly be used as a semi-active system to tune the resonance frequency to the excitation frequency, although the range of variation of the resonance frequency may be too limited to be practical.

This study suggests that energy harvesting using impact should be carefully matched to the excitation available. In particular, harvesting using impact works best when the impacts are intermittent and so the free response of the higher modes is excited; the improvement in harvesting due to the periodic response to periodic excitation is very limited. Hence future work will study situations where the base excitation is random, or systems where free bodies such as balls impact on the beams. Furthermore, the parameters of the energy harvesting systems must be optimised based on the type and bandwidth of the excitation. In particular, the stiffness of the impact (which is a combination of the beam stiffness and the contact stiffness) should be optimised to strongly excite the higher modes.

The authors acknowledge the financial support from the Sêr Cymru National Research Network and Swansea University through a Postgraduate Scholarship NRN-103.

\section{Author contribution statement}

GMA designed the experiment, performed the measurements, and undertook the initial analysis. GMA also wrote the initial simulations of the system. MIF refined the analysis of the measured data, and undertook further simulation studies. HHK and SA provided advice during the experimental and simulation work on the nonlinear dynamics (HHK) and the energy harvesting (SA) aspects. The initial draft of the paper was written by GMA and MIF, and HHK and SA provided critical comments on this draft.

Open Access This is an open access article distributed under the terms of the Creative Commons Attribution License (http://creativecommons.org/licenses/by/4.0), which permits unrestricted use, distribution, and reproduction in any medium, provided the original work is properly cited.

\section{References}

1. A. Erturk, D.J. Inman, Smart Mater. Struct. 18, 025009 (2009)

2. S. Adhikari, M.I. Friswell, D.J. Inman, Smart Mater. Struct. 18, 115005 (2009)

3. S. Kim, W.W. Clark, Q.-M. Wang, J. Intell. Mater. Syst. Struct. 16, 847 (2005)

4. S. Kim, W.W. Clark, Q.-M. Wang, J. Intell. Mater. Syst. Struct. 16, 855 (2005)

5. B. Cavallier, P. Berthelot, H. Nouira, E. Foltete, L. Hirsinger, S. Ballandras, IEEE Ultrasonics Symposium 2, 943 (2005)

6. M. Umeda, K. Nakamura, S. Ueha, Jpn. J. Appl. Phys. 35, 3267 (1996) 
7. M. Renaud, P. Fiorini, C. Van Hoof, Smart Mater. Struct. 16, 1125 (2007)

8. E. Jacquelin, S. Adhikari, M.I. Friswell, Smart Mater. Struct. 20, 105008 (2011)

9. W.J. Stronge, Impact Mechanics (Cambridge University Press, 2004)

10. L. Gu, C. Livermore, Smart Mater. Struct. 20, 045004 (2011)

11. K. Vijayan, M.I. Friswell, H.H. Khodaparast, S. Adhikari, Int. J. Mech. Sci. 96, 101 (2015)

12. G. Martínez-Ayuso, M.I. Friswell, S. Adhikari, H. Haddad Khodaparast, C.A. Featherston, Procedia Eng. 199, 3468 (2017)

13. E.F. Crawley, J. de Luis, AIAA J. 25, 1373 (1987)

14. E.F. Crawley, E.H. Anderson, J. Intell. Mater. Syst. Struct. 1, 4 (1990)

15. C. Park, C. Walz, I. Chopra, Smart Mater. Struct. 5, 98 (1996)

16. O. Bilgen, A. Erturk, D.J. Inman, J. Vib. Acoust. 132, 051005 (2010) 